\title{
Protest dokumentieren
}

\section{Die Sammlung "Neue Soziale Bewegungen" im 21. Jahrhundert}

„Die Bürger sind in Bewegung - nur wie und wohin sie sich bewegen, darüber weiß man noch sehr wenig." ${ }^{1}$ Stuttgart 21, Fridays for Future und

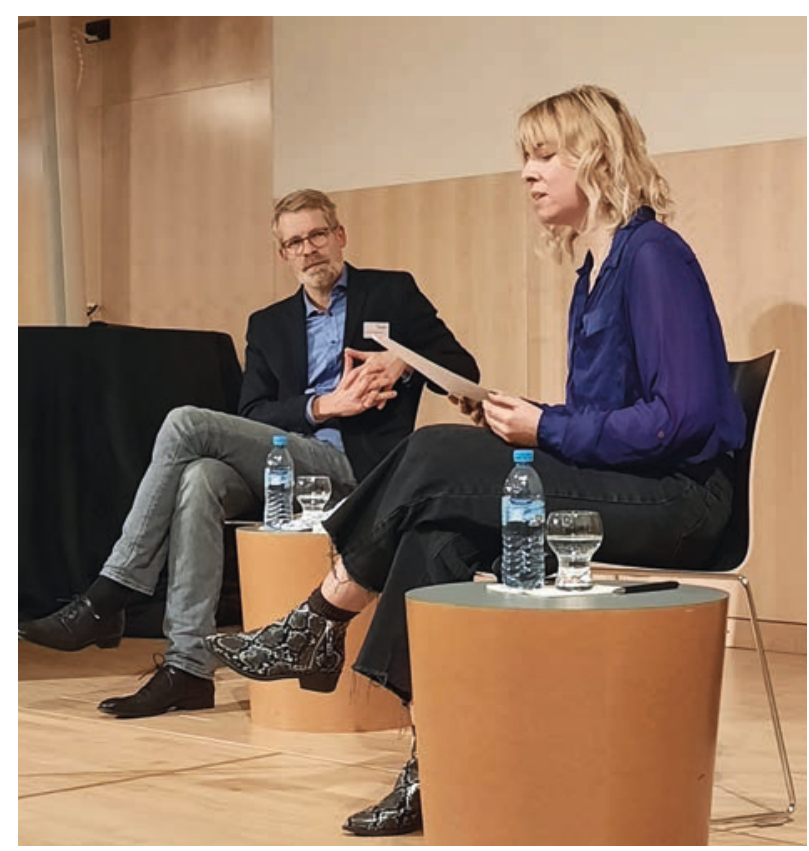

Abb. 1: Christian Westerhoff und Julia von Staden

Anti-Corona: Protestkultur tritt in Stuttgart und anderswo in Erscheinung. Am 22. Oktober 2020 diskutierte Christian Westerhoff im Rahmen der Vortragsreihe der Bibliothek für Zeitgeschichte (BfZ) mit Dr. Julia von Staden und Prof. Dr. Philipp Gassert über die bunte und lebendige Protestkultur, die sich in den letzten Jahren etabliert hat. ${ }^{2}$

Fazit der Gesprächsrunde: In Deutschland wird protestiert. Gegen Stuttgart 21, für mehr Klimaschutz, für bezahlbaren Wohnraum, eine Verkehrswende oder den Erhalt des Hambacher Forstes. Aber auch für den Diesel, gegen Corona-Maßnahmen und gegen die Aufnahme von Geflüchteten. Während lange Zeit vor allem links-alternative und ökologische Bewegungen ihren Unwillen auf die Straße trugen, ist dies heute längst kein Alleinstellungsmerkmal mehr. Protest ist heute auch bürgerlich, konservativ oder rechts. Protestbewegungen kommt in der

1) Walter, Franz: Bürger in Bewegung. Zur Einführung, in: Walter, Franz u.a. (Hg.): Die neue Macht der Bürger. Was motiviert die Protestbewegungen? Reinbek: Rowohlt 2013, S. 9.

2) Die Diskussion ist online einsehbar unter https://lisa.gerda-henkelstiftung.de/bfz_protestkultur
Demokratie eine wichtige Rolle zu: Sie machen auf Probleme aufmerksam und leisten auf diese Weise einen wichtigen Beitrag zur Meinungsbildung. Viele gesellschaftliche Themen und Entwicklungen reizen Bürgerinnen und Bürger, gegen oder für etwas zu demonstrieren. In den letzten Jahren hat sich eine Protestkultur entwickelt, wie sie die Bundesrepublik seit den frühen 1980er-Jahren nicht mehr gesehen hat. Doch woher kommt dieser neue Wille zum Straßenprotest im digitalen Zeitalter? Was macht den Spießbürger zum Wutbürger? Wo und in welcher Form findet heute Protest statt? Und wie versuchen Protestbewegungen auf ihre Anliegen aufmerksam zu machen?

Die neue Protestkultur wirft viele Fragen auf, mit denen sich auch die Wissenschaft intensiv auseinandersetzt. Die Referent*innen der Diskussion am 22. Oktober 2020 hoben hervor, dass es für die Forschung essentiell sei, dass die Aktivitäten von Protestbewegungen dokumentiert und deren zunehmend digitale Kommunikation archiviert werden. Außerdem scheint es sich bei vielen Erscheinungen im Protestgeschehen um neue Entwicklungen zu handeln; tatsächlich gab es viele Phänomene wie z.B. die Bildung einer Gegenöffentlichkeit auch schon in der Vergangenheit. In anderen Bereichen, z.B. beim Umgang der Polizei mit Protestierenden, wurde aus der Geschichte gelernt. Daher ist es vielfach hilfreich, einen Blick auf die langen Entwicklungen zu werfen.

\section{Weiterentwicklung der Sammlung}

Mit Blick auf die große Bedeutung des Themas Protest plant die BfZ, ihre Sammlung "Neue Soziale Bewegungen" neu aufzustellen. Im Auftrag der Deutschen Forschungsgemeinschaft (DFG) sammelte die BfZ seit 1972 Medien der 68er-Bewegung und der aus ihr hervorgegangenen gesellschaftlichen Strömungen und Gruppierungen. Zum Sammelspektrum "Neue Soziale Bewegungen" zählten neben der Studentenbewegung und der Außerparlamentarischen Opposition (APO) die Friedensbewegung, die Anti-Atomkraft-Bewegung, die 
Dritte-Welt-Bewegung, das Engagement für Menschen- und Bürgerrechte, Initiativen zu den Themen Migration, Ausländer und Flüchtlinge sowie der Rechts- und Linksradikalismus und der damit in Verbindung stehende Terrorismus. Es entstand ein umfangreicher Bestand von ca. 138.000 Flugblättern, 27.500 Plakaten, 20.000 Broschüren und 1.000 Zeitschriften und Zeitungen zur alternativen politischen Kultur der Bundesrepublik. Leider wurde die Sammeltätigkeit im Jahr 2005 eingestellt.
Materialien ab dem Jahr 2005 bis heute. Neben Broschüren, Flugblättern und Plakaten erweist sich das Sammeln von Nachlässen und Akten als sinnvolle Ergänzung. Da Protest ein sichtbares Produkt eines Austauschprozesses sowohl innerhalb der protestierenden Gruppe als auch mit deren Umwelt ist, erscheint die Erweiterung der Sammeltätigkeit um diese Dokumente umso bedeutsamer. Interesse gilt zudem Beständen aus privaten Händen bzw. von kleinen Einrichtungen, die nicht selbst für

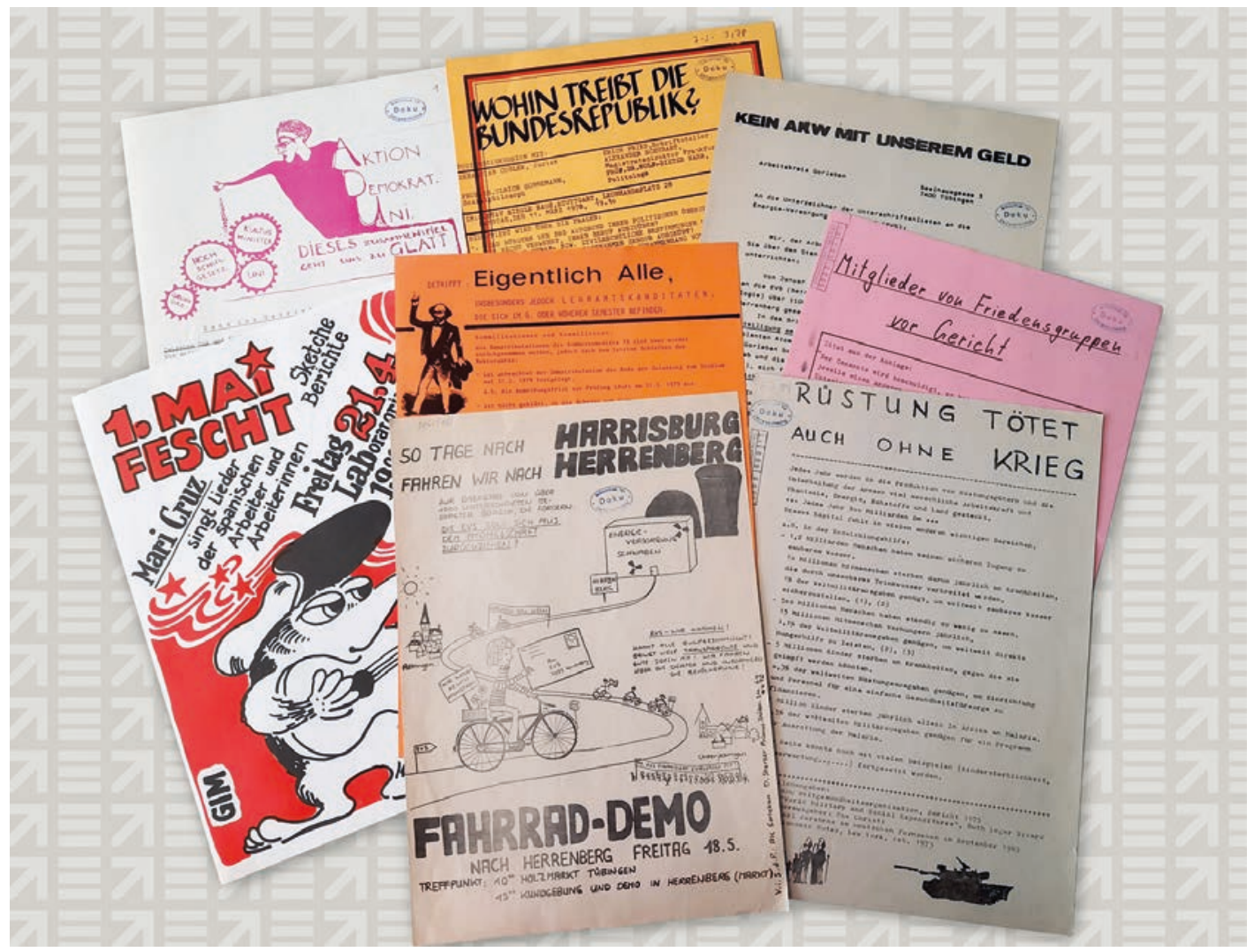
eine professionelle Aufarbeitung und Archivierung sorgen können. Materialien sollen sowohl in digitaler als auch in analoger Form zu aktuellen Protesten zusammengetragen werden. Zwar werden während Demonstrationen nach wie vor Transparente hochgehalten und Flugblätter verteilt, jedoch ist der digitale Wandel auch in diesem Teil der sozialen Lebenswelt prä-

Abb. 2: Flugblätter der Sammlung "Neue Soziale Bewegungen"

In Anbetracht des bereits existierenden Bestands und vorhandener bibliothekarischer sowie fachwissenschaftlicher Kompetenzen entwickelt die BfZ aktuell Pläne zur Wiederaufnahme der Sammeltätigkeit. Dies soll in enger Zusammenarbeit mit Wissenschaftler*innen, die zum Themengebiet Soziale Bewegungen und Protest forschen, erfolgen. Eine genauere Betrachtung zeigt, dass diese Thematik nicht nur von den Politik- oder Geschichtswissenschaften erforscht wird, sondern auch von den Fachbereichen Soziologie, Kultur-, Medien- und Kommunikationswissenschaften. Der zukünftige Aufbau der Sammlung muss dabei die Interessen einzelner Disziplinen sowie interdisziplinärer Ansätze berücksichtigen. In den Fokus rücken sent. Die meisten Organisationen haben eigene Internetseiten, die als Hauptinformationsquelle über die Bewegung gelten. Dort wird über die Ziele und aktuelle Aktionen der Bewegung informiert, rechtliche und organisatorische Tipps zu Veranstaltungen gegeben, Broschüren, Flugblätter und Plakate zum Herunterladen und Ausdrucken bereitgestellt, Pressespiegel und eigene Pressemitteilungen veröffentlicht. Auch über diverse Social-Media-Kanäle ( $z$. B. Facebook, Twitter, Youtube, Instagram) werden die Einladungen zu Demonstrationen und Widerstandsaktionen sowie die Berichterstattungen von Aktionen geteilt. Wenn wir uns die „Flüchtigkeit des Internets" vor Augen halten, ist die Ausweitung der Sammeltätigkeit auf die digitalen Quellen in Form der Webarchivierung unabdingbar. 
Diese konkrete Wiederaufnahme der Sammeltätigkeit geht mit Arbeiten einher, die es zum Ziel haben, den Bestand für die Forschung stärker zugänglich zu machen. Die enge Zusammenarbeit mit Forscher*innen im Bereich der Protestbewegungen bringt der BfZ nicht nur Impulse bei der Planung dieses Vorhabens, sondern soll ebenso zur regen Nutzung der bisher gesammelten Materialien beitragen. Um der Forschung die Quellen zur Verfügung zu stellen, wurden bereits einzelne Schritte unternommen:

- 20.000 Flugblätter, insbesondere aus BadenWürttemberg, sind online recherchierbar (erschlossen in Allegro-Datenbank). ${ }^{3}$

- große Teile der Zeitschriften (mit Ausnahme von Einzelheften) und Broschüren sind erschlossen und über den Bibliothekskatalog ${ }^{4}$ recherchierbar.

- ein Teil der Plakate (ca. 500 von 27.500) ist für die Nutzer zugänglich (erschlossen in AllegroDatenbank). ${ }^{5}$

- aktuell wird die Digitalisierung des Bestands von 138.000 Flugblättern geplant.
Bereits Mitte der 1990er-Jahre erkannte Niklas Luhmann: "Stärker noch als das Bekenntnis zur sozialen Marktwirtschaft und zu ihrer demokratischen Dividende hat die Gewohnheit zu protestieren einen festen Platz in der Geschichte der Bundesrepublik". ${ }^{6}$ Die Bedeutung der Neuen Sozialen Bewegungen wurde schon Anfang der 1970er-Jahre von der DFG wahrgenommen und führte zur Finanzierung des Sondersammelgebiets bei der Bibliothek für Zeitgeschichte. Auch in den letzten Jahren ist die Zahl der Proteste und der damit in Verbindung stehenden Bewegungen deutlich gewachsen. Aus diesen Gründen ist die Wiederaufnahme der Sammeltätigkeit, die nicht nur vergangene, sondern auch aktuelle Protestbewegungen dokumentiert, besonders wichtig.

Beata Lakeberg / Christian Westerhoff 